\title{
Radiomics Methods to Differentiate Metastasis and Primary Lung Cancer of Breast Cancer Patients in PET/CT
}

Xinglu Zhou ( $\square$ zhouxinglu@hrbmu.edu.cn )

Harbin Medical University Cancer Hospital

\section{Yunsong Peng}

University of Science and Technology of China

\section{Yingci Li}

Harbin Medical University Cancer Hospital

Jiarui Zhang

Second Affiliated Hospital of Harbin Medical University

Tianyi Liu

Second Affiliated Hospital of Harbin Medical University

\section{Huijie Jiang}

Second Affiliated Hospital of Harbin Medical University

Jian Zheng

University of Science and Technology of China

\section{Research Article}

Keywords: breast cancer, PET/CT, radiomics, second primary lung cancer

Posted Date: June 2nd, 2021

DOI: https://doi.org/10.21203/rs.3.rs-502469/v1

License: (c) (1) This work is licensed under a Creative Commons Attribution 4.0 International License. Read Full License 
Title: Radiomics methods to differentiate metastasis and primary lung cancer of breast cancer patients in $\mathrm{PET} / \mathrm{CT}$

Xinglu Zhou ${ }^{1,2}$ : zhouxinglu@hrbmu.edu.cn

Yunsong Peng ${ }^{3,4}:$ pys@mail.ustc.edu.cn

Yingci Li': Ivliyingci@163.com

Jiarui Zhang5: 1013010135@qq.com

Tianyi Liu6: 109146440@qq.com

Huijie Jiang²: jianghuijie@hrbmu.edu.cn

Jian Zheng ${ }^{3,4}: \underline{\text { zhengj@sibet.ac.cn }}$

1. Department of PET/CT Center, Harbin Medical University Cancer Hospital, Harbin 150081, China

2. Department of Radiology, Second Affiliated Hospital of Harbin Medical University, Harbin 150086, China

3. School of Biomedical Engineering (Suzhou), Division of Life Sciences and Medicine. University of Science and Technology of China, Hefei 230026, China

4. Department of Medical Imaging, Suzhou Institute of Biomedical Engineering and Technology, Chinese Academy of Sciences, Suzhou 215163, China

5. Department of Breast Surgery, Second Affiliated Hospital of Harbin Medical University, Harbin 150086, China

6. Department of Pathology, Second Affiliated Hospital of Harbin Medical University, Harbin 150086, China 


\section{Abstract}

Purpose: Positron emission tomography (PET) with integrated computed tomography (PET/CT) is a whole-body imaging method providing information the entire body. When it was used in staging breast cancer patients, quite a few patients were found to have a second primary lung cancer(PLC), which was has few distinguishing features from breast cancer metastasis(MBC). Therefore, based on CT, LDCT and PET images, combined with pathological features, we established radiomics models to distinguish between MBC and PLC.

Methods: We retrospectively collected CT, LDCT, and PET images, and pathology features of 100 breast cancer patients, including 60 metastases of breast cancer(MBC) and 40 primary lung cancers(PLC). The two radiologists manually drew a region of interest around the whole visible tumor in consensus. Python 3.8 and Pyradiomics toolkit are used to extract features from CT, LDCT, and PET. The linear discriminant analysis (LDA) classifier was used to build the radiomics model. The receiver operating characteristic (ROC) curve and area under the ROC curve (AUC) were used to evaluate the classification performance.

Results: Total 12, 13, and 9 features were selected from the CT, LDCT, and PET respectively. The model based on the LDCT and PET obtained the same highest AUC (0.9479). The combination with CT and pathology features showed a highest AUC of 0.9583 with a sensitivity of 1.000 and a specificity of 0.8333 .

Conclusion: Overall, the results are encouraging that radiomics models based on CT, LDCT and PET can differentiate between MBC and PLC pathological features could significantly improve the AUC and ACC of CT model.

Keywords: breast cancer, $\mathrm{PET} / \mathrm{CT}$, radiomics, second primary lung cancer

\section{Introduction}

Breast cancer, as the most common malignant tumor in women, greatly harms women's health. Treatment of breast cancer has progressed extraordinarily with the introduction of more and more personalized therapies ${ }^{[1]}$. Although major advances have been made in diagnosis and treatment of primary breast cancer, metastasis, typically to bone, lung, liver, and brain, is still the leading reason for mortality of cancer patients ${ }^{[2]}$. The lung is often the first site of metastasis for $\mathrm{BC}$, especially among the clinically aggressive basal subtype of breast cancer ${ }^{[3]}$. A increasing opportunity for the development of second caners since the better survival is a matter of course. By 10 years after diagnosis, approximately $10 \%$ of breast cancer survivors have developed a subsequent malignancy ${ }^{[4]}$. Lung cancer accounts for approximately $5 \%$ of second primary cancers among breast cancer survivors. Therefore, the identification of metastatic breast cancer patients $(M B C)$ and primary lung cancer $(P L C)$ is crucial in clinic practice, because $M B C$ is the malignant lung tumor in its advanced stage ${ }^{[5]}$. According to several studies, there were significant 
differences between the PLC and MBC with respect to overall survival, disease-free survival or recurrence control ${ }^{[6,]}$. The presentation of lung lesions in patients with breast cancer warrants a comprehensive evaluation of primary lung cancer and metastatic breast cancers, because diagnosis and resection at an early stage is associated with improved survival[Error! Bookmark not defined.]. Therefore, there is a need for imaging techniques that can predict PLC and MBC of breast cancer patients.

Fluorine-18-fluorodeoxyglucose $\left({ }^{18} \mathrm{~F}-\mathrm{FDG}\right)$ positron emission tomography (PET) with integrated computed tomography (PET/CT) is not only combining functional and anatomical imaging within a single examination in a hybrid technique, but also providing information of the entire body in one imaging method. It has became a very sensitive and accurate technique for tumor diagnosis and staging. LDCT has emerged as a promising mass screening method for the early diagnosis of lung neoplasms ${ }^{[8,9]}$ since its simplicity and its high sensitivity ${ }^{[10,11]}$. Thus, PET/CT and CT scans are the preferred modality for lung nodule no matter whether it's a metastasis or a primary cancer of the breast cancer patient. And current guidelines on breast cancer recommend that the staging evaluation of women who present with breast cancer includes PET/CT and LDCT ${ }^{[12,13]}$.

However, in the actual diagnosis process, morphological features of CT and PET/CT are not specific enough to differentiate between PLC and MBC, there are certain limitations in the diagnosis. Several studies have reported that radiomics may help distinguish benign and malignant pulmonary nodules ${ }^{\text {[Error! Bookmark not defined.,14] }}$ or detecting lung metastases in patients with cancers ${ }^{[15,16]}$ based on CT and PET. And there were researches on the ability of PET and CT radiomics features to differentiate between primary and metastatic lung lesions ${ }^{[17,18]}$. However, the radiomics approach has not yet been tested in the differential diagnosis between PLC and MBC. Therefore, the main aims of our study is to evaluate the ability of PET and CT-based radiomics to predict the probability that a breast cancer patient with lung lesions has a primary lung cancer or a metastasis.

\section{Materials and Method}

Patients

In this retrospective single-center investigation, we collected 158 breast cancer or breast cancer history patients with lung lesions who were examined by FDG PET/CT during the 14 days before the biopsy or surgical procedure between 1 January 2011 and November 302020 at Harbin Medical University Tumor Hospital. The inclusion criteria were: histological diagnosis from CT-guided biopsy or surgical removal of a lung lesion. The exclusion criteria were: (a) inconclusive histology from an inadequate biopsy sample, (b) diagnosis of nonmalignancy, and (c) the area of interest cannot be delineated. In the end, 60 breast cancer patients with lung metastasis, 40 breast cancer patients with lung cancer, a total of 100 cases were included in this study. In our work, all patients were divided into two cohorts to build the predictive model and evaluate the prediction results. The patients collected from January 2011 to July 2019 were used as the 
training set $(n=80)$, and patients collected from August 2019 to November 2020 were used as the validation set $(n=20)$. All procedures performed in studies involving human participants were in accordance with the ethical standards of Harbin Medical University. Appropriate informed consent for the retrospective investigation was obtained from each patient.

Histopathologic Assessment

One pathologist with 15 years of experience in pathology assessed the histologic results of breast lesions and lung lesions. The histologic type and grade were evaluated from histopathologic reports of US-guided core biopsies or surgery. The expression of estrogen receptor, progesterone receptor, human epidermal growth factor 2 (HER2), and Ki-67 was assessed by using the standard avidinbiotin complex immunohistochemical staining method. Estrogen receptor and progesterone receptor positivity were assessed with the Allred score, which rates the proportion of positive cells (on a scale of 0-5) and the staining intensity (on a scale of 0-3). Tumors were considered estrogen receptor or progesterone receptor positive if the Allred score exceeded 3. Tumors were considered HER2 positive if they had a score of $3+$ at immunohistochemical examination. If HER2 status was equivocal (score, 2+) at immunohistochemical examination, fluorescence in situ hybridization analysis was performed to confirm the diagnosis ${ }^{[19]}$.

\section{FDGPET/CT and low-dose CT}

All patients were fasted for $6 \mathrm{~h}$ and blood glucose levels were required to be $<10 \mathrm{mmol} / \mathrm{l}$ before scanning by using an integrated PET/CT scanner (Discovery ST: GE Medical systems, Milwaukee, WI, USA). All PET images were corrected for attenuation using the acquired CT data. The tube voltage is $120 \mathrm{kV}$, the tube current is $200 \mathrm{mAs}$, and the slice thickness is $3.75 \mathrm{~mm}$. PET collection uses 3D mode PET scanning, $2.5 \mathrm{~min} /$ bed, and generally scans $6-8$ beds. Image recombination reconstructs images using the ordered subset maximum expectation method. Low-dose CT(LDCT) was performed directly after receiving $\mathrm{PET} / \mathrm{CT}$ scan with parameters of $120 \mathrm{kv}$ tube voltage, $50 \mathrm{mAs}$ tube current, and $1.00 \mathrm{~mm}$ slice thickness. All ${ }^{18} \mathrm{~F}$-FDG PET/CT and LDCT images were reviewed by two experienced senior physicians who were aware of the patient's clinical history and laboratory results.

The volume of interest (VOI)

Data management was performed by two independent radiologists (radiologists A and B) with 6 and 12 years of experience in PET/CT diagnosis. Both radiologists had access to all PET/CT images to assist in locating the lesions and verifying the lesion boundaries. The two radiologists manually drew a region of interest around the whole visible tumor in consensus by ITK-SNAP 3.8.0 (as shown in figure 1). If the cancer was multifocal or multicentric, the region of interest was measured at the tumor with the largest size. The Intra-class correlation coeffificients (ICCS) were 
adopted to evaluate the intra- and inter-observer agreement of the extracted features. An ICC greater than 0.6 are considered to meet the criteria for feature reproducibility, and greater than 0.8 are considered tobe almost perfect agreement. Radiologist $A$ drew the ROls twice at two-month intervals, and radiologist B drew the ROIs independently according to the same tumor boundary definition. Good intra- and inter-observer agreement was achieved with an ICC range of 0.691-0.995.

\section{Feature Extraction}

Python 3.8 and Pyradiomics toolkit ${ }^{[20]}$ are used to extract features from CT, LDCT, and PET. For each modality, a total of 851 features, including first-order, shape, texture, and wavelet features, were extracted. The first order features show the image intensity knowledge, the shape features reflect the morphological characteristics of lesions, and the texture features describe the texture changes inside and outside the lesions. Note that, the wavelet features include all the above features except shape features, which were extracted from the wavelet filtered images of the original image. The clinical features were ER, PR, HER-2, and Ki67. All the image features are shown in Table 1.

\section{Feature Selection}

To avoid overfitting, the feature selection method from coarse to fine was adopted. First, the highly correlated features were grouped by the Spearman correlation test $(|\rho| \geqslant 0$. 95), and a representative feature was selected from each group with the highest AUC value. Second, the least absolute shrinkage and selection operator algorithm (LASSO) ${ }^{[21]}$ was used to select the most discriminative features by the largest AUC value in 10 times of 10 -fold cross-validation in training set with the LASSO-Logistic regression model.

\section{Radiomics model}

The linear discriminant analysis (LDA) classifier ${ }^{[22]}$ was used to build the radiomics model. The LDA classifier was constructed by the key features from the training set and evaluated in the independent testing set. To evaluate the performance of models, the AUC, accuracy, sensitivity, and specificity had been calculated. We used features from each modality (CT, LDCT, PET, and clinical information) to build single-modality models. At the same time, we also tried to build models based on fusions of each imaging modality with clinical features, which were $\mathrm{CT}+\mathrm{Clinical}$ model, LDCT+Clinical model, and PET+Clinical model. 
The SPSS software, version 21 (SPSS, Chicago, IL, USA), was used for the statistical analyses. The normality of the variable distribution was validated by the Kolmogorov-Smirnov test. The Student's t-test or Mann-Whitney $U$ test was used to compare the statistical differences of quantitative variables, when appropriate. The chi-square test was used to compare the statistical differences of qualitative variables. All statistical tests were two-sided and $p$ values less than 0.05 were considered statistically significant.

\section{Results}

Patient characteristics

The study cohort included 100 breast cancer patients, including 60 metastases of breast cancer(MBC) and 40 primary lung cancers(PLC). The mean age of $M B C$ was $53.7 \pm 7.2$ years, range from 32-77. The mean age of PLC was 60.5 \pm 5.4 years, range from 39-82.

\section{Lung Lesions Characteristics}

The greatest dimension of the MBC was $2.2 \pm 1.4 \mathrm{~cm}$ (range $0.6-5.8 \mathrm{~cm}$ ) and that of the PLC was $2.7 \pm 1.5 \mathrm{~cm}$ (range1.3-9.2cm). The median SUVmax value of the MBC was $8.7 \pm 5.6$ (rang 1.4-26.8) and that of the PLC was 7.9 \pm 6.0 (range 1.6-24.0). There was not a statistically significant difference between the greatest dimension $(p=0.154)$ and SUVmax $(p=0.568)$ of MBC and primary lung cancer.

\section{Pathology Characteristics}

The pathology of breast cancer in $60 \mathrm{MBC}$ patients were 57 cases of invasive ductal carcinoma, one metaplastic squamous cell carcinoma, one mucinous carcinoma, and one invasive micropapillary carcinoma. All these 60 patients included 34(56.7\%) cases of ER positive, 21(35.0\%) cases of PR positive, $11(18.3 \%)$ cases of HER-2 positive and $49(81.7 \%)$ cases of ki-67 positive.The pathology of breast cancer in 40 PLC patients were 31 invasive ductal carcinomas, 8 noninvasive ductal carcinomas, and one mucinous carcinoma.The immunophenotypes of breast cancer in these 40 patients included 29(72.5\%) cases of ER positive, 27(67.5\%) cases of PR positive, $11(27.5 \%)$ cases of HER-2 positive and $23(57.5 \%)$ cases of ki-67 positive. All 40 primary lung cancers were histologically confirmed as 2 squamous carcinomas, one small cell carcinoma and 37 adenocarcinomas. Patient characteristics are listed in Table 1.

\section{Selected Features}

A total of 851 fetaures including first-order, shape, texture, and wavelet features, were extracted from CT, LDCT, and PET as listed in Table2. Regarding the features of the optimal radiomics signature model, 34 features were selected, including 1 first-order features and 33 wavelet features shown in Table 3. Through feature selection, 12, 13, and 9 features were selected from the CT, LDCT, and PET respectively. Except for 1 first-order feature selected for LDCT, all the other 
33 features were derived from wavelet features for CT, LDCT, and PET. Shape feature and texture feature were not selected in all models. The mean,median, and variance values of the 34 selected features are also listed in Table 4. Furthermore, 10 of 12 features from CT, 10 of 13 features from LDCT, and all 9 features from PET were significantly different between metastasis of breast cancer and primary lung cancer $(P<0.05)$.

The accuracy, sensitivity, specificity, and ROC analysis results from 7 feature sets are shown and compared in figure 2 and Table 5. The model based on the LDCT and PET obtained the same highest AUC (0.9479) among the four single-sequence models. Then the 3 imaging features combined with pathology features separately were modeled as two-sequence models. The combination with CT and clinical features showed a highest AUC of 0.9583 with a sensitivity of 1.000 and a specificity of 0.8333 . The combination with LDCT and pathology features also showed a higher AUC of 0.9375 , a sensitivity of 0.75 , and a specificity of 1.0 (shown in figure 3 ).

\section{Discussion}

This study attempted to noninvasively distinguish lung metastasis and primary lung cancer of bresat cancer patients using a CT, LDCT, and PET radiomics features model with pathology features of primary breast cancer, which demonstrated a promising diagnostic performance in an independent validation set.

According to the AUCs, the models of the 3 individual radiomics features sequences all showed reasonable diagnostic efficiency, and the features extracted from LDCT and PET contributed the most to the differentiation of lung lesions. As previous research proved that LDCT has a very good ability in the identification of lung lesions $\left.{ }^{[E r r o r ! ~ B o o k m a r k ~ n o t ~ d e f i n e d, ~} 23,24\right]$. And in our study, LDCT has a thinner scan thickness, and its resolution is higher than that of CT and PET images, so it can display more details, and extracting an modeling better of radiomics features.

${ }^{18}$ FDG-PET quantified by standardized uptake values (SUV), has been mostly applied in functional imaging form for clinical practices ${ }^{[25,26]}$. It is generally known to be strongly related to Glucose-transporter family (GLUT)-expression in tumors, which convey the glucose uptake into cells. There is an moderate relatedness between GLUT 1 expression and SUV values derived from

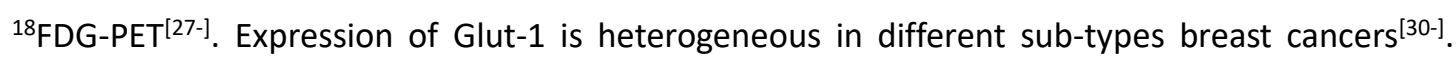
Thus, the degree of 18FDG uptake seems to be more heterogeneous in comparison with many other cancers, which has been the main reason for the present investigation of the causal mechanism that determines ${ }^{18} \mathrm{FDG}$ uptake in breast cancer ${ }^{[33]}$. Present available literature recommends that the use of ${ }^{18} \mathrm{~F}-\mathrm{FDG}$ PET and PET/CT leads to significant modification of staging and treatment in diagnosed breast cancer patients ${ }^{[34-36]}$. Therefore, PET imaging may be applied for clinical routine use in the breast cancer initial staging. On the other hand, it can find an 
unexpected second primary cancer ${ }^{[37-]}$, among which lung cancer is one of the most common primary cancers of breast cancer patients ${ }^{[0,41]}$. Lung cancer, especially lung adenocarcinoma, which is the largest number of lung cancer types in our study, also has certain heterogeneity in FDG uptake and SUVmax values ${ }^{[42]}$. As another aspect, it was exemplarily shown for lung cancer SUVmax seems to be related to vessel density in tissues ${ }^{[43,44]}$. In addition, whether they are the $\mathrm{MBC}$ or the second PLC, they are all malignant tumors, although studies have shown that the SUVmax value of metastatic lesions in the lung is slightly higher than that of the primary cancer ${ }^{[45,46]}$. Considering that the research object in our study is breast cancer. We can see that

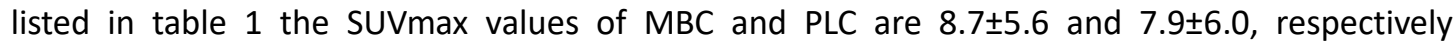
$(p=0.568)$, that were not statistically significant. As shown in figure 4 , the SUVmax of MBC and PLC are 3.2 and 2.8. Therefore, ${ }^{18} \mathrm{FDG}$-PET is necessary for breast cancer patients, but it could not be used to distinguish between MBC and PLC along.

Not only that, due to the heterogeneity of breast cancer, the appearance of its metastases on CT is also very different. Therefore, as shown in figure 4 , we can see metastases with rough edges or with a density of ground glass. These findings are similar to the imaging findings of primary lung adenocarcinoma ${ }^{[47]}$. At the same time, in our results, we did not select tumor size, shape and texture features, because these features are not helpful to our model. Therefore, when radiologists distinguish between MBC and PLC solely through CT images, it may produce some diagnostic bias.

Radiomics has the potential to uncover disease characteristics by extracting numerous parameters/features from tomographic images within a region of interest using mathematical algorithms ${ }^{[48]}$. In our research, except for one first-order feature in LDCT, the other 33 features are all wavelet features. The wavelet features obtained through wavelet transformation of the image can obtain image features that are difficult to obtain from the original image ${ }^{[49-]}$. In previous studies, radiomics analysis based on wavelet features has been widely used in the identification, prognostic analysis, and elaboration of therapeutic protocols of lung lesions ${ }^{[52-]}$, and it has a good predictive ability. Therefore in our study, the single models based on wavelet features of CT, LDCT, and PET also have high accuracy, and the AUC is 0.8854, 0.9479, and 0.9479 respectively. This is similar to the research results of other scholars(from 0.71 to 0.98 ), and even better than some research models ${ }^{[56-]}$.

Among the pathology features, PR and Ki67 have certain significance in identifying lung lesions . In MBC, PR- and KI-67+ have a larger number(65.00\% and $81.67 \%)$, which is consistent with the conclusion of previous scholars that PR- and $\mathrm{Ki}-67+$ have high propensities to metastasis ${ }^{[60]}$ (shown in figure 5). However, there is still no clear conclusion on which type or subtype of breast cancer patients are prone to develop second primary lung cancer. For example, earlier studies believed that radiotherapy after breast cancer can significantly increase the incidence of lung cancer ${ }^{[61,62]}$. It was also found in studies that ER- or triple-negative breast cancer patients are more likely to develop lung cancer particularly during the first 5 years after 
breast cancer diagnosis ${ }^{[63,64]}$. But in our study, although there is no statistical difference, there are more ER+ numbers in PLC patients, there were 29(72.50\%) ER+ in PLC, and 34(56.67\%) ER+ in $\mathrm{MBC}$ as listed in table 1 and shown in figure 6 . The results seem to be contradictory, but due to the deviation of the research subjects, the results are not comparable. The cohort of this study was breast cancer patients with lung lesions, so it cannot be said that patients with PR+ and KI-67- are more likely to get second primary lung cancer. Therefore, the clinical, pathological and genetic characteristics that affect the occurrence of PLC need to be further studied.

When we added pathological features to CT model, we found that both AUC(0.9583) and ACC(0.9) were significantly improved, but on the contrary, when pathological features were added to the LDCT and PET models, both AUC and ACC did not increase, and even had a small range decrease. The reason may be that pathological features add some unnecessary features to LDCT and PET whose AUC are already very high, which increases the noise of the data, thereby increasing the error of the learning algorithm. The AUC of the CT single model is lower. After adding pathological features, its diagnostic performance is significantly improved, and it is higher than the AUC of the LDCT and PET single models. Therefore, we believe that pathological information will play a certain role in the optimization of the model, but the correlation between pathology and radiomics features is not the main content of this study, and further research is needed to discuss the relationship between pathological and radiomics features of lung lesions among breast cancer patients.

\section{Limitations}

With regard to limitations of our study, firstly, there may be selection bias because the subjects only consisted of 100 patients, and a radiomics study with a larger sample size is still needed in the future. Secondly only 4 pathological features of the primary breast cancer were included in this study to distinguish between MBC and PLC. Although a better model is obtained, in future research, more clinical and pathological information needs to optimize the models. Thirdly, segmentation were not automatic or semi-automatic, but we have manually segmented VOI 3 times by two radiologists to minimize the doctor's supervisor deviation.

\section{Conclusion}

In this study, a radiomics method based on pathological features, LDCT and PET/CT data was proposed for the classification for PLC and MBC of breast cancer patients. The features extracted from LDCT and PET contributed the most to the differentiation of PLC and MBC. Pathological features could significantly improve the AUC and ACC of CT model.

\section{Acknowledge}


Financial supported from Harbin Medical University Cancer Hospital Haiyan Fund Youth Funding Project (no. JJQN2020-13) and Scientific Research Project of Heilongjiang Health Commission (no.2020-073) were acknowledged.

The experimental protocol was approved by Harbin Medical University ethics committee. Consent for publication was obtained from all the participants/ patients.

1 ]] De Abreu FB, Schwartz GN, Wells WA, Tsongalis GJ. Personalized therapy for breast cancer: Personalized therapy for breast cancer. Clin Genet. 2014;86(1):62-67. doi:10.1111/cge.12381

2 [] Luck AA, Evans AJ, Green AR, Rakha EA, Paish C, Ellis IO. The influence of basal phenotype on the metastatic pattern of breast cancer. Clin Oncol (R Coll Radiol). 2008;20(1):40-45. doi:10.1016/j.clon.2007.10.002

3 [] Paget S. The distribution of secondary growths in cancer of the breast. 1889. Cancer Metastasis Rev. 1989;8(2):98-101.

4 [] Hayat MJ, Howlader N, Reichman ME, Edwards BK. Cancer statistics, trends, and multiple primary cancer analyses from the Surveillance, Epidemiology, and End Results (SEER) Program. Oncologist. 2007;12(1):20-37. doi:10.1634/theoncologist.12-1-20

5 [] Asamura H. Multiple primary cancers or multiple metastases, that is the question. J Thorac Oncol. 2010;5(7):930-931. doi:10.1097/JTO.0b013e3181e15d8c

6 [?] Kerendi F, Gal A, Corvera JS, Halkos ME, Miller JI. Characteristics of second primary lung malignancy in patients with known breast cancer. South Med J. 2009;102(3):269-274. doi:10.1097/SMJ.0b013e318197fec6

7 国] Kaufman EL, Jacobson JS, Hershman DL, Desai M, Neugut Al. Effect of breast cancer radiotherapy and cigarette smoking on risk of second primary lung cancer. J Clin Oncol. 2008;26(3):392-398. doi:10.1200/JC0.2007.13.3033

8 [] Becker N, Motsch E, Trotter A, et al. Lung cancer mortality reduction by LDCT screening-Results from the randomized German LUSI trial. Int J Cancer. 2020;146(6):1503-1513. doi:10.1002/ijc.32486

9 [] National Lung Screening Trial Research Team, Aberle DR, Adams AM, et al. Reduced lung-cancer mortality with low-dose computed tomographic screening. $\mathrm{N}$ Engl J Med. 2011;365(5):395-409. doi:10.1056/NEJMoa1102873

10 [?] Seijo LM, Peled N, Ajona D, et al. Biomarkers in Lung Cancer Screening: Achievements, Promises, and Challenges. J Thorac Oncol. 2019;14(3):343-357. doi:10.1016/j.jtho.2018.11.023 
11 [?] Smith RA, Andrews KS, Brooks D, et al. Cancer screening in the United States, 2019: A review of current American Cancer Society guidelines and current issues in cancer screening. CA Cancer J Clin. 2019;69(3):184-210. doi:10.3322/caac.21557

12 [?] 1. Cardoso F, Kyriakides S, Ohno S, et al. Early breast cancer: ESMO Clinical Practice Guidelines for diagnosis, treatment and follow-up. Annals of Oncology. 2019;30(8):1194-1220. doi:10.1093/annonc/mdz173

13 [?] Gradishar WJ, Anderson BO, Abraham J, et al. Breast Cancer, Version 3.2020, NCCN Clinical Practice Guidelines in Oncology. J Natl Compr Canc Netw. 2020;18(4):452-478. doi:10.6004/jnccn.2020.0016

14 ?] Ruilong Z, Daohai X, Li G, Xiaohong W, Chunjie W, Lei T. Diagnostic value of 18F-FDG-PET/CT for the evaluation of solitary pulmonary nodules: a systematic review and meta-analysis. Nucl Med Commun. 2017;38(1):67-75. doi:10.1097/MNM.0000000000000605

15 ?] Ferreira-Junior JR, Koenigkam-Santos M, Magalhães Tenório AP, et al. CT-based radiomics for prediction of histologic subtype and metastatic disease in primary malignant lung neoplasms. Int J Comput Assist Radiol Surg. 2020;15(1):163-172. doi:10.1007/s11548-019-02093-y

16 ?] Qi Y, Cui X, Han M, et al. Radiomics analysis of lung CT image for the early detection of metastases in patients with breast cancer: preliminary findings from a retrospective cohort study. Eur Radiol. 2020;30(8):4545-4556. doi:10.1007/s00330-020-06745-5

17 ?] Kirienko M, Cozzi L, Rossi A, et al. Ability of FDG PET and CT radiomics features to differentiate between primary and metastatic lung lesions. Eur J Nucl Med Mol Imaging. 2018;45(10):1649-1660. doi:10.1007/s00259-018-3987-2

18 ?] Zhou Y, Ma XL, Zhang T, Wang J, Zhang T, Tian R. Use of radiomics based on 18F-FDG PET/CT and machine learning methods to aid clinical decision-making in the classification of solitary pulmonary lesions: an innovative approach [published online ahead of print, 2021 Feb 5]. Eur J Nucl Med Mol Imaging. 2021;10.1007/s00259-021-05220-7. doi:10.1007/s00259-021-05220-7

19 ?] Xue H C , Wang L, Jian L I, et al. Expression of estrogen receptor,progesterone receptor and human epidermal growth factor receptor 2 in breast cancer stem cells and their differentiated cells[J]. Journal of Xinxiang Medical University, 2017.

20 [?] van Griethuysen JJM, Fedorov A, Parmar C, et al. Computational Radiomics System to Decode the Radiographic Phenotype. Cancer Res. 2017;77(21):e104-e107. doi:10.1158/0008-5472.CAN-17-0339

21 [?] Tibshirani, R. "Regression Shrinkage and Selection via the Lasso." Journal of the Royal Statistical Society. Series B, Vol. 58, No. 1, 1996, pp. 267-288. 
22 [?] Riffenburgh R H, Clunies-Ross C W . Linear Discriminant Analysis. Chicago, 2013, $3(6): 27-33$.

23 ?] Liu J, Xu H, Qing H, et al. Comparison of Radiomic Models Based on Low-Dose and Standard-Dose CT for Prediction of Adenocarcinomas and Benign Lesions in Solid Pulmonary Nodules. Front Oncol. 2021;10:634298. Published 2021 Feb 2. doi:10.3389/fonc.2020.634298

24 []] Wang YH, Chen CF, Lin YK, Chiang C, Tzao C, Yen Y. Predicting malignancy: subsolid nodules detected on LDCT in a surgical cohort of East Asian patients. J Thorac Dis. 2020;12(8):4315-4326. doi:10.21037/jtd-20-659

25 ?] Garcia-Velloso MJ, Bastarrika G, de-Torres JP, et al. Assessment of indeterminate pulmonary nodules detected in lung cancer screening: Diagnostic accuracy of FDG PET/CT. Lung Cancer. 2016;97:81-86. doi:10.1016/j.lungcan.2016.04.025

26 ?] Han S, Choi JY. Impact of 18F-FDG PET, PET/CT, and PET/MRI on Staging and Management as an Initial Staging Modality in Breast Cancer: A Systematic Review and Meta-analysis. Clin Nucl Med. 2021;46(4):271-282. doi:10.1097/RLU.0000000000003502

27 ?] Meyer HJ, Wienke A, Surov A. Associations between GLUT expression and SUV values derived from FDG-PET in different tumors-A systematic review and meta analysis. PLoS One. 2019;14(6):e0217781. Published 2019 Jun 17. doi:10.1371/journal.pone.0217781

28 ?] Kitajima K, Miyoshi Y, Yamano T, Odawara S, Higuchi T, Yamakado K. Prognostic value of FDG-PET and DWI in breast cancer. Ann Nucl Med. 2018;32(1):44-53. doi:10.1007/s12149-017-1217-9

29 [?]Chen X, Lu P, Zhou S, Zhang L, Zhao JH, Tang JH. Predictive value of glucose transporter-1 and glucose transporter-3 for survival of cancer patients: A meta-analysis. Oncotarget. 2017;8(8):13206-13213. doi:10.18632/oncotarget.14570

30 ?] Choi J, Kim DH, Jung WH, Koo JS. Metabolic interaction between cancer cells and stromal cells according to breast cancer molecular subtype. Breast Cancer Res. 2013;15(5):R78. doi:10.1186/bcr3472

31 [?] Gowrishankar G, Zitzmann-Kolbe S, Junutula A, et al. GLUT 5 is not over-expressed in breast cancer cells and patient breast cancer tissues. PLoS One. 2011;6(11):e26902. doi:10.1371/journal.pone.0026902

32 ?] Shi $Y$, Zhang $Y$, Ran F, et al. Let-7a-5p inhibits triple-negative breast tumor growth and metastasis through GLUT12-mediated warburg effect. Cancer Lett. 2020;495:53-65. doi:10.1016/j.canlet.2020.09.012

33 ?]] Avril N, Rosé CA, Schelling $M$, et al. Breast imaging with positron emission tomography and fluorine-18 fluorodeoxyglucose: use and limitations. J Clin Oncol. 2000;18(20):3495-3502. 
doi:10.1200/JCO.2000.18.20.3495

34 [] Ozer N, Sahin A. Correlation of Breast Cancer Subgroups and Axillary Metastases with 18F-FDG PET/CT and the Contribution of 18F-FDG PET/CT in the Management of the Axilla. J Coll Physicians Surg Pak. 2021;31(2):150-155. doi:10.29271/jcpsp.2021.02.150

35 [?] Nakajima H, Maeno K, Ito T, et al. Concomitant use of 18F-FDG PET-CT SUVmax is useful in the assessment of Ki67 labeling index in core-needle biopsy specimens of breast cancer. Gland Surg. 2021;10(1):1-9. doi:10.21037/gs-20-485

36 []] Dehdashti F, Wu N, Ma CX, Naughton MJ, Katzenellenbogen JA, Siegel BA. Association of PET-based estradiol-challenge test for breast cancer progesterone receptors with response to endocrine therapy. Nat Commun. 2021;12(1):733. Published 2021 Feb 2. doi:10.1038/s41467-020-20814-9

37 [] Kikuchi $M$, Shinohara $S$, Hino $M$, et al. Detection of subclinical recurrence or second primary cancer using (18) F-FDG PET/CT in patients treated curatively for head and neck squamous cell carcinoma. Head Neck. 2016;38 Suppl 1:E511-E518. doi:10.1002/hed.24032

38 [] Xu H, Zhang M, Zhai G, Li B. The clinical significance of 18F-FDG-PET/CT in early detection of second primary malignancy in cancer patients. J Cancer Res Clin Oncol. 2010;136(8):1125-1134. doi:10.1007/s00432-010-0759-1

39 [] Chen H, Zhao L, Ruan D, Sun L, Lin Q. 68Ga-FAPI PET/CT Improves Therapeutic Strategy by Detecting a Second Primary Malignancy in a Patient With Rectal Cancer. Clin Nucl Med. 2020;45(6):468-470. doi:10.1097/RLU.0000000000003000

40 []] Kaufman EL, Jacobson JS, Hershman DL, Desai M, Neugut Al. Effect of breast cancer radiotherapy and cigarette smoking on risk of second primary lung cancer. J Clin Oncol. 2008;26(3):392-398. doi:10.1200/JCO.2007.13.3033

41 [] Lorigan $\mathrm{P}$, Califano R, Faivre-Finn C, Howell A, Thatcher N. Lung cancer after treatment for breast cancer. Lancet Oncol. 2010;11(12):1184-1192. doi:10.1016/S1470-2045(10)70056-5

42 []] Peng L, Feng L, Yuan H, Benhabbour SR, Mumper RJ. Development of a novel orthotopic non-small cell lung cancer model and therapeutic benefit of 2'-(2-bromohexadecanoyl)-docetaxel conjugate nanoparticles. Nanomedicine. 2014;10(7):1497-1506. doi:10.1016/j.nano.2014.03.016

43 [] Koh YW, Lee SJ, Park SY. 18F-fluorodeoxyglucose positron emission tomography is correlated with the pathological necrosis and decreased microvessel density in lung adenocarcinomas. Ann Nucl Med. 2019;33(2):93-102. doi:10.1007/s12149-018-1309-1

44 []] Surov A, Meyer HJ, Wienke A. Standardized Uptake Values Derived from 18F-FDG PET May Predict Lung Cancer Microvessel Density and Expression of KI 67, VEGF, and HIF-1 $\alpha$ but Not Expression of Cyclin D1, PCNA, EGFR, PD L1, and p53. Contrast Media Mol Imaging. 2018;2018:9257929. Published 2018 Jun 6. doi:10.1155/2018/9257929 
45 [] Kosaka N, Tsuchida T, Tsuji K, Shimizu K, Kimura H. Standardized uptake value differences between primary and metastatic lesions in 18F-FDG PET/CT of patients with lung cancer. Acta Radiol. 2015;56(11):1329-1335. doi:10.1177/0284185114556705

46 []] Liu Y, Tang Y, Xue Z, et al. SUVmax Ratio on PET/CT May Differentiate Between Lung Metastases and Synchronous Multiple Primary Lung Cancer. Acad Radiol. 2020;27(5):618-623. doi:10.1016/j.acra.2019.07.001

47 [] Satoh H, Ishikawa H. Pre-invasive lung adenocarcinoma lesions on CT. Clin Respir J. 2018;12(1):354. doi:10.1111/crj.12473

48 [] Gillies RJ, Kinahan PE, Hricak H. Radiomics: Images Are More than Pictures, They Are Data. Radiology. 2016;278(2):563-577. doi:10.1148/radiol.2015151169

49 [?] Wang ZZ, Yong JH. Texture analysis and classification with linear regression model based on wavelet transform. IEEE Trans Image Process. 2008;17(8):1421-1430. doi:10.1109/TIP.2008.926150

50 [?] Dubey SR, Singh SK, Singh RK. Local Wavelet Pattern: A New Feature Descriptor for Image Retrieval in Medical CT Databases. IEEE Trans Image Process. 2015;24(12):5892-5903. doi:10.1109/TIP.2015.2493446

51 []] Pun CM, Lee MC. Extraction of shift invariant wavelet features for classification of images with different sizes. IEEE Trans Pattern Anal Mach Intell. 2004;26(9):1228-1233. doi:10.1109/TPAMI.2004.67

52 [?] Thawani R, McLane $M$, Beig $N$, et al. Radiomics and radiogenomics in lung cancer: $A$ review for the clinician. Lung Cancer. 2018;115:34-41. doi:10.1016/j.lungcan.2017.10.015

53 [] Madero Orozco H, Vergara Villegas OO, Cruz Sánchez VG, Ochoa Domínguez Hde J, Nandayapa Alfaro Mde J. Automated system for lung nodules classification based on wavelet feature descriptor and support vector machine. Biomed Eng Online. 2015;14:9. Published 2015 Feb 12. doi:10.1186/s12938-015-0003-y

54 [?] Depeursinge A, Yanagawa M, Leung AN, Rubin DL. Predicting adenocarcinoma recurrence using computational texture models of nodule components in lung CT [published correction appears in Med Phys. 2015 May;42(5):2653]. Med Phys. 2015;42(4):2054-2063. doi:10.1118/1.4916088

55 [?] Starkov P, Aguilera TA, Golden DI, et al. The use of texture-based radiomics CT analysis to predict outcomes in early-stage non-small cell lung cancer treated with stereotactic ablative radiotherapy. Br J Radiol. 2019;92(1094):20180228. doi:10.1259/bjr.20180228

56 [?] Zhang R, Zhu L, Cai Z, et al. Potential feature exploration and model development based on 18F-FDG PET/CT images for differentiating benign and malignant lung lesions. Eur J Radiol. 2019;121:108735. doi:10.1016/j.ejrad.2019.108735 
57 [] Feng B, Chen $X$, Chen $Y$, et al. Radiomics nomogram for preoperative differentiation of lung tuberculoma from adenocarcinoma in solitary pulmonary solid nodule. Eur J Radiol. 2020;128:109022. doi:10.1016/j.ejrad.2020.109022

58 [?] Sun $Y$, Li C, Jin L, et al. Radiomics for lung adenocarcinoma manifesting as pure ground-glass nodules: invasive prediction. Eur Radiol. 2020;30(7):3650-3659. doi:10.1007/s00330-020-06776-y

59 []] Kang F, Mu W, Gong J, et al. Integrating manual diagnosis into radiomics for reducing the false positive rate of 18F-FDG PET/CT diagnosis in patients with suspected lung cancer. Eur J Nucl Med Mol Imaging. 2019;46(13):2770-2779. doi:10.1007/s00259-019-04418-0

60 [] Kast K, Link T, Friedrich K, et al. Impact of breast cancer subtypes and patterns of metastasis on outcome. Breast Cancer Res Treat. 2015;150(3):621-629. doi:10.1007/s10549-015-3341-3

61 [?] Henson KE, McGale P, Taylor C, Darby SC. Radiation-related mortality from heart disease and lung cancer more than 20 years after radiotherapy for breast cancer. $\mathrm{Br} J$ Cancer. 2013;108(1):179-182. doi:10.1038/bjc.2012.575

62 [?] Grantzau T, Overgaard J. Risk of second non-breast cancer among patients treated with and without postoperative radiotherapy for primary breast cancer: A systematic review and meta-analysis of population-based studies including 522,739 patients. Radiother Oncol. 2016;121(3):402-413. doi:10.1016/j.radonc.2016.08.017

63 [] Schonfeld SJ, Curtis RE, Anderson WF, Berrington de González A. The risk of a second primary lung cancer after a first invasive breast cancer according to estrogen receptor status. Cancer Causes Control. 2012;23(10):1721-1728. doi:10.1007/s10552-012-0054-3

64 [] Wang R, Yin Z, Liu L, et al. Second Primary Lung Cancer After Breast Cancer: A Population-Based Study of 6,269 Women. Front Oncol. 2018;8:427. Published 2018 Oct 9. doi:10.3389/fonc.2018.00427

1 [] Kitajima K, Miyoshi Y, Yamano T, Odawara S, Higuchi T, Yamakado K. Prognostic value of FDG-PET and DWI in breast cancer. Ann Nucl Med. 2018;32(1):44-53. doi:10.1007/s12149-017-1217-9

2 [?] Gowrishankar G, Zitzmann-Kolbe S, Junutula A, et al. GLUT 5 is not over-expressed in breast cancer cells and patient breast cancer tissues. PLoS One. 2011;6(11):e26902. doi:10.1371/journal.pone.0026902

3 []] Nakajima $\mathrm{H}$, Maeno $\mathrm{K}$, Ito T, et al. Concomitant use of 18F-FDG PET-CT SUVmax is useful in the assessment of Ki67 labeling index in core-needle biopsy specimens of breast cancer. Gland Surg. 2021;10(1):1-9. doi:10.21037/gs-20-485 
4 [] $\mathrm{Xu} \mathrm{H}$, Zhang $\mathrm{M}$, Zhai G, Li B. The clinical significance of 18F-FDG-PET/CT in early detection of second primary malignancy in cancer patients. J Cancer Res Clin Oncol. 2010;136(8):1125-1134. doi:10.1007/s00432-010-0759-1

5 []] Dubey SR, Singh SK, Singh RK. Local Wavelet Pattern: A New Feature Descriptor for Image Retrieval in Medical CT Databases. IEEE Trans Image Process. 2015;24(12):5892-5903. doi:10.1109/TIP.2015.2493446

6 [] Madero Orozco H, Vergara Villegas OO, Cruz Sánchez VG, Ochoa Domínguez Hde J, Nandayapa Alfaro Mde J. Automated system for lung nodules classification based on wavelet feature descriptor and support vector machine. Biomed Eng Online. 2015;14:9. Published 2015 Feb 12. doi:10.1186/s12938-015-0003-y

7 [?] Depeursinge A, Yanagawa M, Leung AN, Rubin DL. Predicting adenocarcinoma recurrence using computational texture models of nodule components in lung CT [published correction appears in Med Phys. 2015 May;42(5):2653]. Med Phys. 2015;42(4):2054-2063. doi:10.1118/1.4916088

8 [?] Feng $B$, Chen $X$, Chen $Y$, et al. Radiomics nomogram for preoperative differentiation of lung tuberculoma from adenocarcinoma in solitary pulmonary solid nodule. Eur J Radiol. 2020;128:109022. doi:10.1016/j.ejrad.2020.109022

9 [?] Sun Y, Li C, Jin L, et al. Radiomics for lung adenocarcinoma manifesting as pure ground-glass nodules: invasive prediction. Eur Radiol. 2020;30(7):3650-3659. doi:10.1007/s00330-020-06776-y

[1] Kitajima K, Miyoshi Y, Yamano T, Odawara S, Higuchi T, Yamakado K. Prognostic value of FDG-PET and DWI in breast cancer. Ann Nucl Med. 2018;32(1):44-53.

doi:10.1007/s12149-017-1217-9

[2] Gowrishankar G, Zitzmann-Kolbe S, Junutula A, et al. GLUT 5 is not over-expressed in breast cancer cells and patient breast cancer tissues. PLoS One. 2011;6(11):e26902.

doi:10.1371/journal.pone.0026902

[3] Nakajima H, Maeno K, Ito T, et al. Concomitant use of 18F-FDG PET-CT SUVmax is useful in the assessment of Ki67 labeling index in core-needle biopsy specimens of breast cancer. Gland Surg. 2021;10(1):1-9. doi:10.21037/gs-20-485

[4] Xu H, Zhang M, Zhai G, Li B. The clinical significance of 18F-FDG-PET/CT in early detection of second primary malignancy in cancer patients. J Cancer Res Clin Oncol. 2010;136(8):1125-1134. doi:10.1007/s00432-010-0759-1

[5] Dubey SR, Singh SK, Singh RK. Local Wavelet Pattern: A New Feature Descriptor for Image Retrieval in Medical CT Databases. IEEE Trans Image Process. 2015;24(12):5892-5903. doi:10.1109/TIP.2015.2493446

[6] Madero Orozco H, Vergara Villegas OO, Cruz Sánchez VG, Ochoa Domínguez Hde J, Nandayapa Alfaro Mde J. Automated system for lung nodules classification based on wavelet feature descriptor and support vector machine. Biomed Eng Online. 2015;14:9. Published 2015 
Feb 12. doi:10.1186/s12938-015-0003-y

[7] Depeursinge A, Yanagawa M, Leung AN, Rubin DL. Predicting adenocarcinoma recurrence using computational texture models of nodule components in lung $\mathrm{CT}$ [published correction appears in Med Phys. 2015 May;42(5):2653]. Med Phys. 2015;42(4):2054-2063.

doi:10.1118/1.4916088

[8] Feng B, Chen X, Chen Y, et al. Radiomics nomogram for preoperative differentiation of lung tuberculoma from adenocarcinoma in solitary pulmonary solid nodule. Eur J Radiol.

2020;128:109022. doi:10.1016/j.ejrad.2020.109022

[9] Sun Y, Li C, Jin L, et al. Radiomics for lung adenocarcinoma manifesting as pure ground-glass nodules: invasive prediction. Eur Radiol. 2020;30(7):3650-3659. doi:10.1007/s00330-020-06776-y 

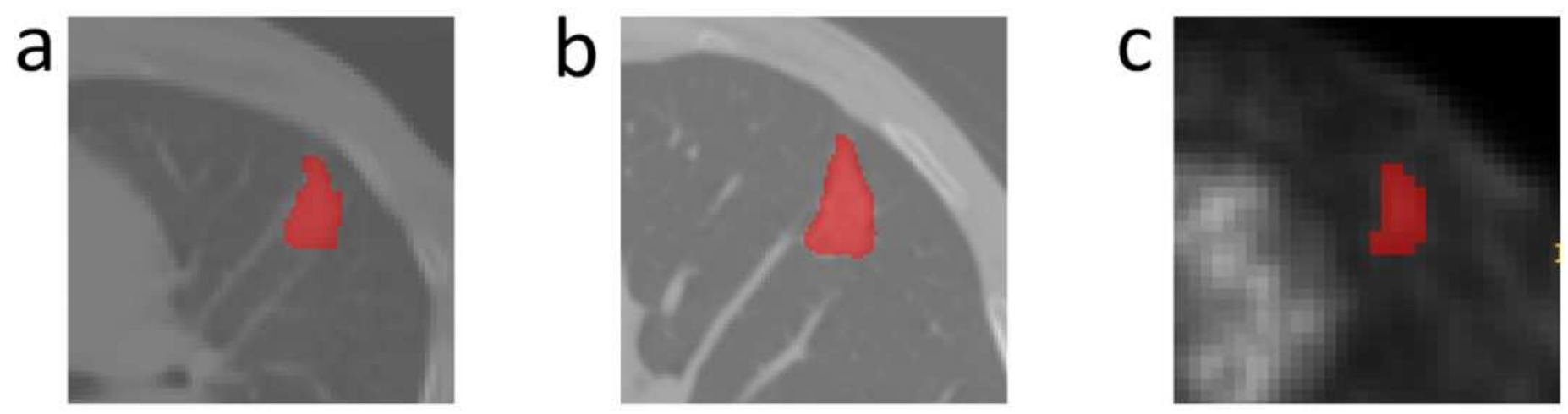

\section{Figure 1}

Example segmentations. A 61-year-old breast cancer patient was diagnosed with lung cancer. Segmentation (red masks) of the tumor overlaid on the original images in CT, LDCT, and PET.

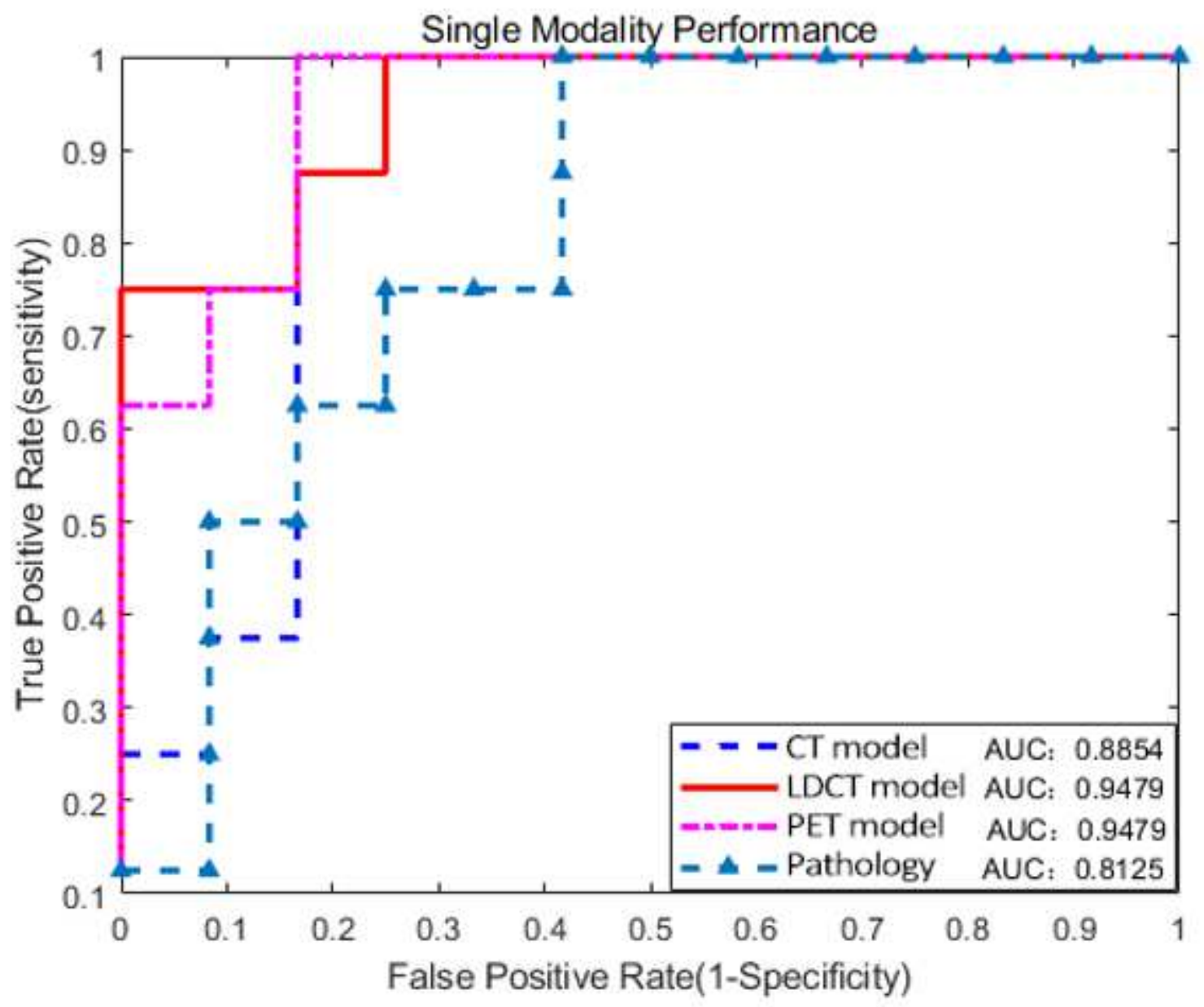

Figure 2 
ROC curve of single radiomics signature models for distinguishing MBC and PLC.

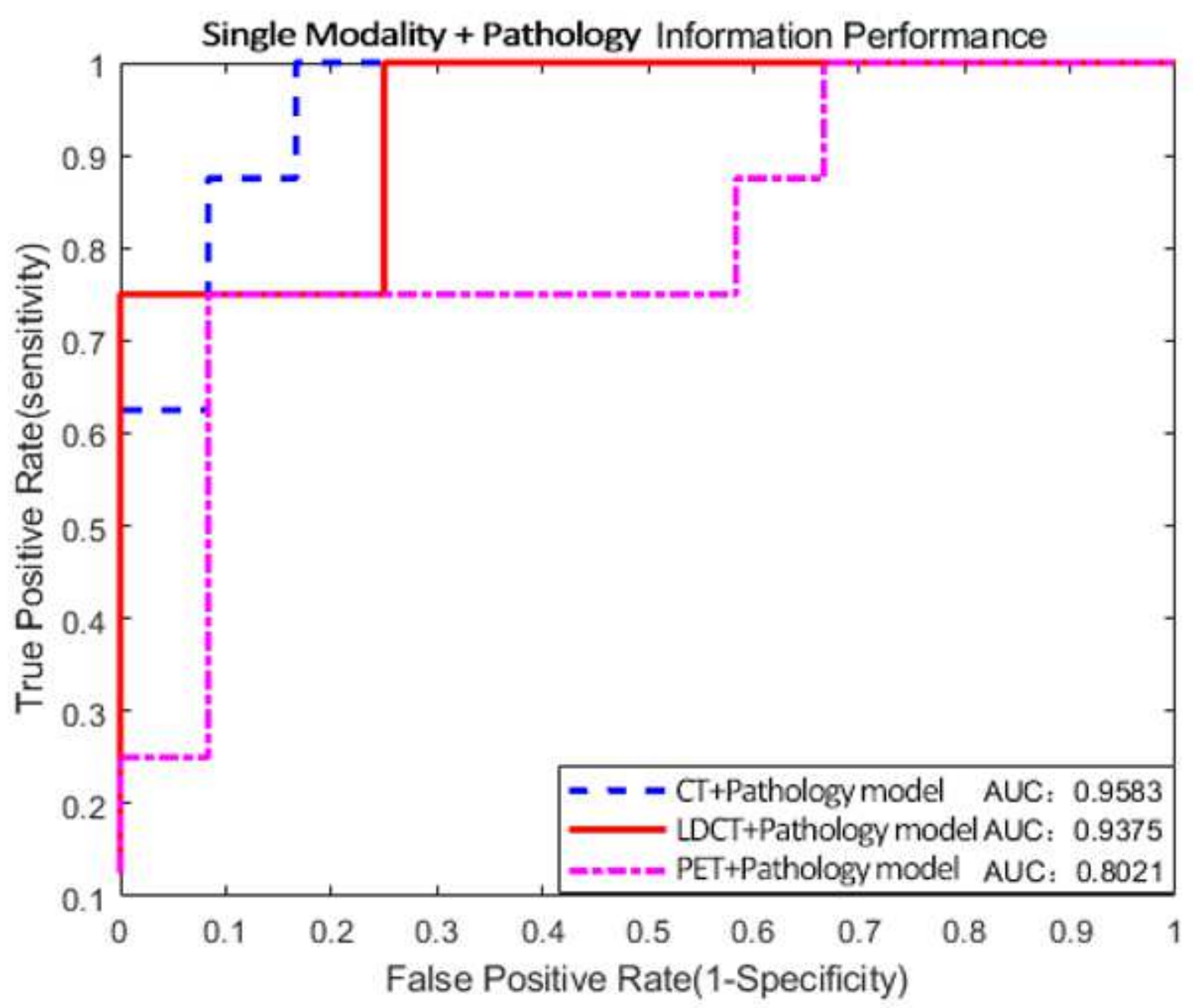

Figure 3

ROC curve of single radiomics signature and pathology models for distinguishing MBC and PLC. 

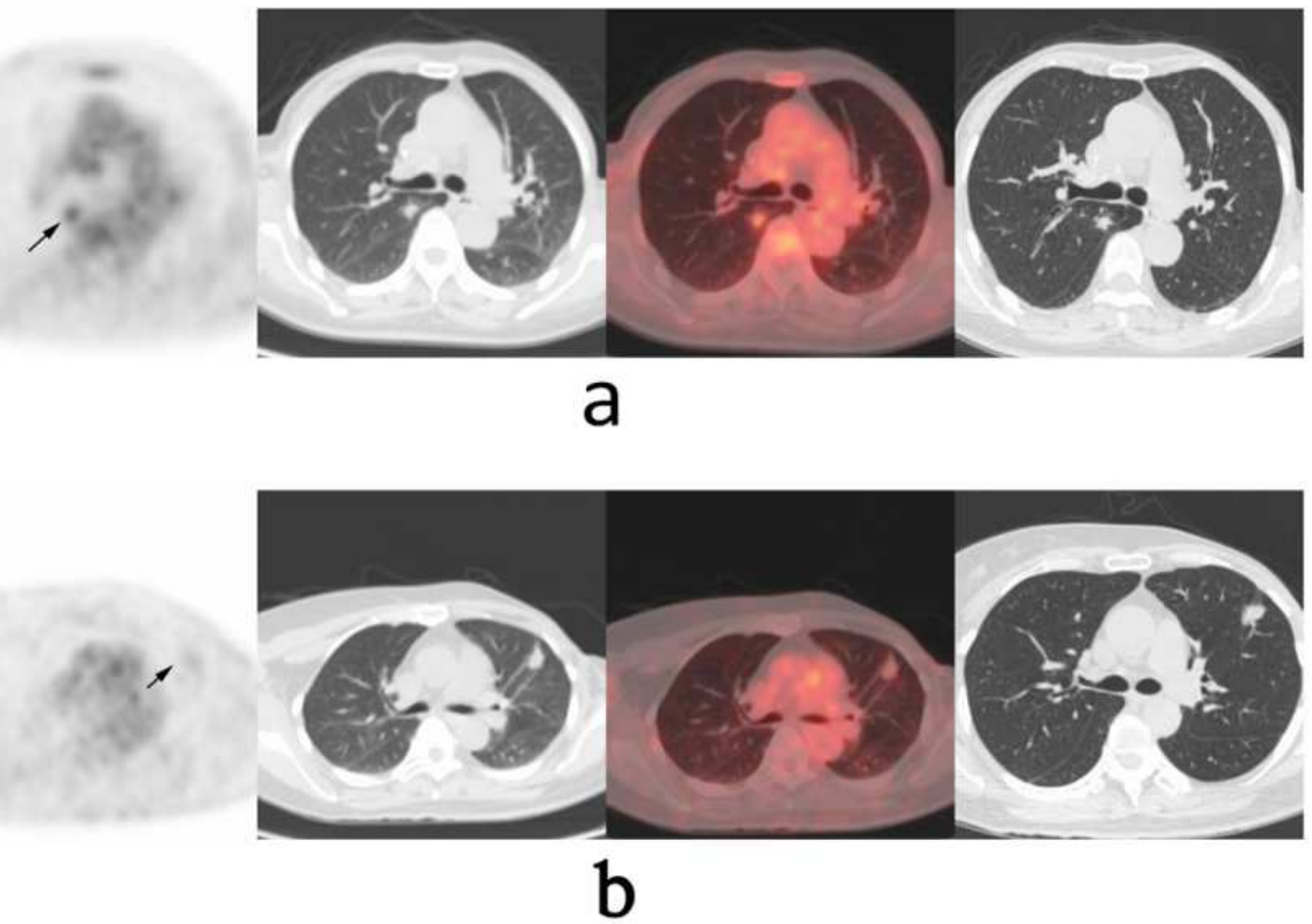

\section{Figure 4}

Imaging of MBC and PLC. (a) A 54-year-old woman with lung metastasis after 5 years diagnosed with double breast cancers; (b) A 61-year-old breast cancer patient diagnosed with lung cancer.

a

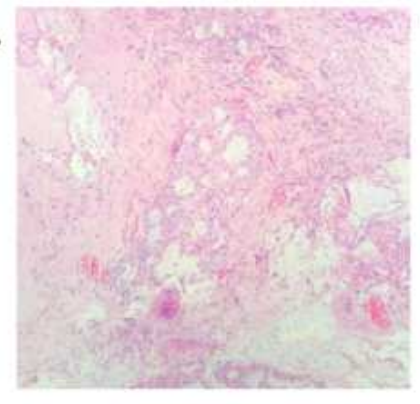

e

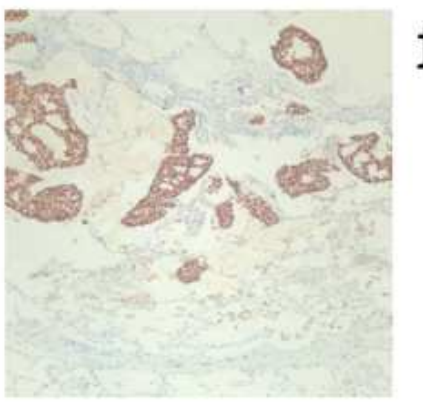

b

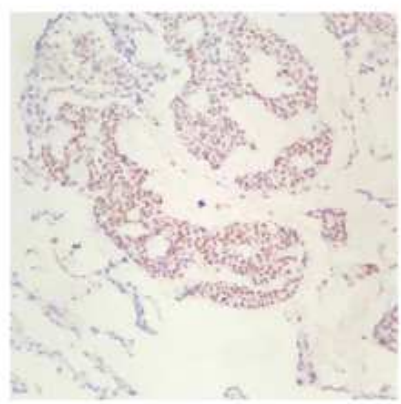

f

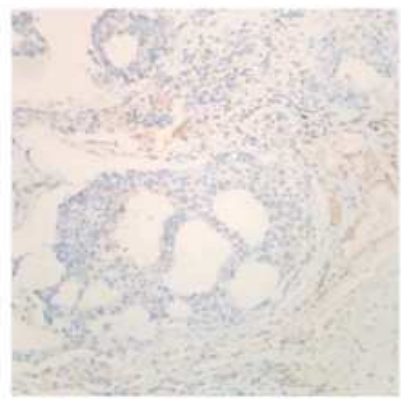

C

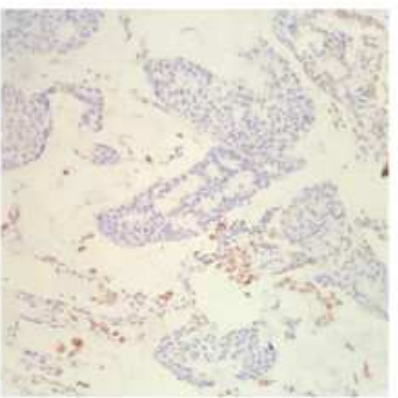

g

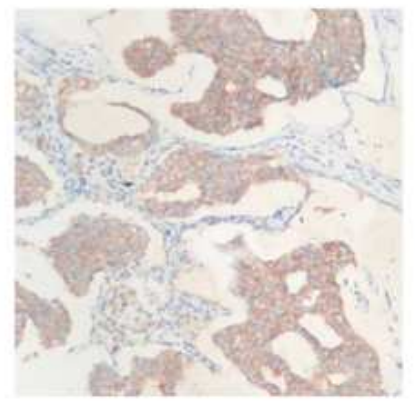

d

h

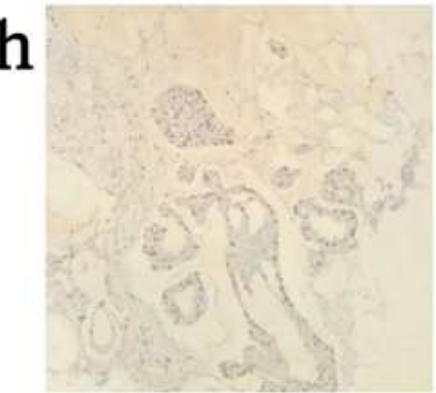




\section{Figure 5}

Example of MBC pathology. A 54-year-old woman with lung metastasis after 5 years diagnosed with double breast cancers (a) HE for lung lesion; (b) GATA-3 positive; (c) Napsin A negative; (d) TTF-1 negative; (e) ER positive; (f) PR negative; (g) Her-2 positive; (h) Ki-67 positive.
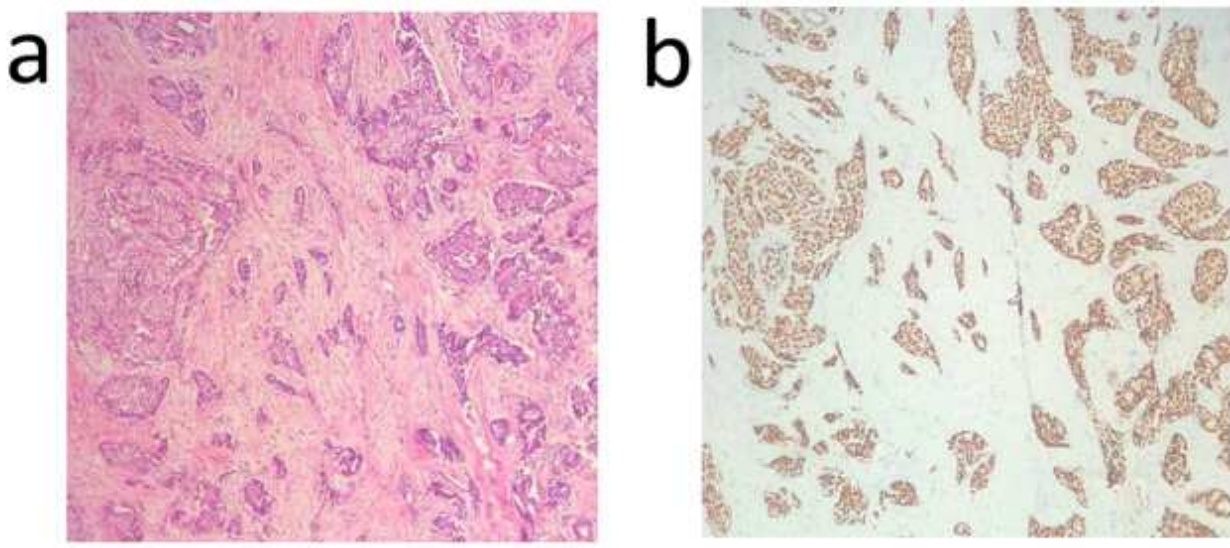

d

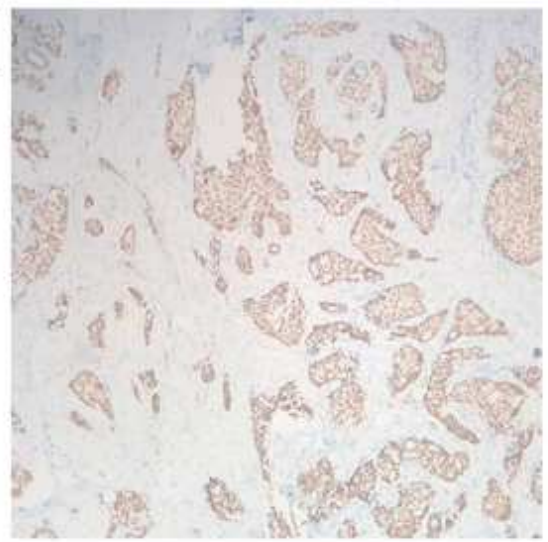

e

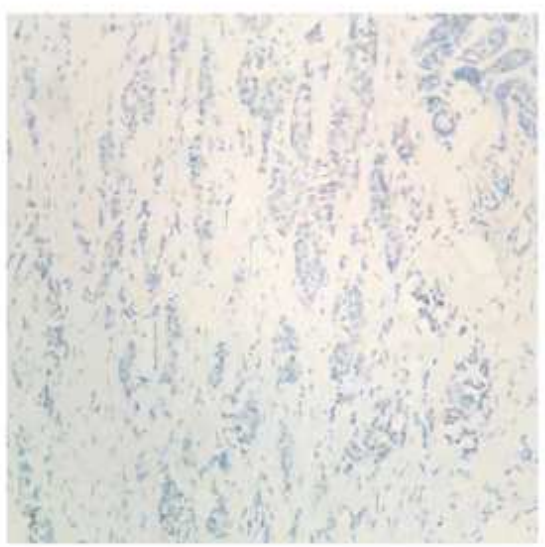

C

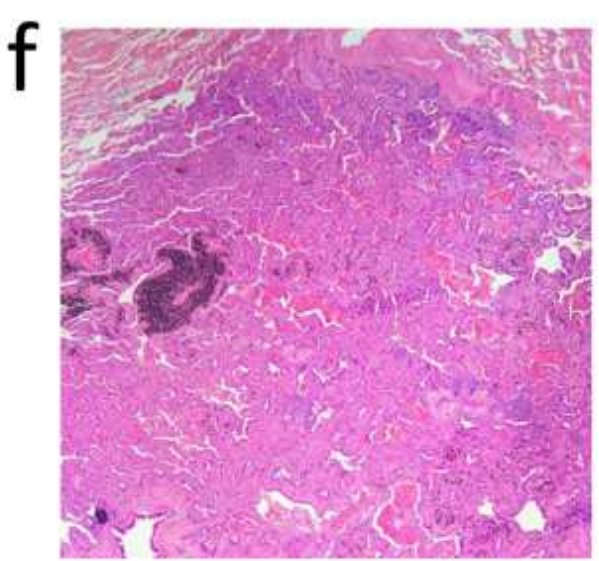

\section{Figure 6}

Example of PLC pathology. A 61-year-old breast cancer patient diagnosed with lung cancer. (a) HE of breast lesion; (b) ER positive; (c) PR positive; (d) Her-2 negative; (d) Ki-67 negative; (f) HE of lung lesion diagnosed with primary lung cancer. 